ФГБУ «Научный медицинский исследовательский центр эндокринологии» Минздрава России, Москва, Россия

В представленном обзоре рассматриваются основные причины недостаточной обеспеченности витамином D населения Российской Федерации, а также приводятся данные о распространенности дефицита витамина D среди различных групп населения и в разных регионах, которые подтверждают повсеместную распространенность дефицита витамина D в стране. Обсуждаемые исследования свидетельствуют о том, что наблюдаемая в настоящее время недостаточная обеспеченность витамином D населения РФ (сниженные уровни 25(OH)D выявляются в среднем у - 80\% лиц в общей популяции) обусловлена как низким уровнем его эндогенного синтеза, так и недостаточным поступлением с пищей: территория страны расположена в зоне низкой инсоляции, и в то же время в рационе населения очень ограничены как основные естественные источники витамина D (морская рыба жирных сортов), так и фортифицированные витамином D продукты. Принятие мер по повышению статуса витамина D и поддержанию оптимальной концентрации 25(OH)D в крови у детского и взрослого населения, адекватное потребление витамина D и при показаниях - его добавок, позволит улучшить состояние костно-мышечной системы, а также возможно снизить риск развития некоторых хронических заболеваний.

КЛЮЧЕВЫЕ СЛОВА: Витамин D; рекомендованная суточная норма; дефицит витамина D; эпидемиология

\title{
THE PREVALENCE OF VITAMIN D DEFICIENCY IN RUSSIAN FEDERATION
}

\author{
(c) Alexandra A. Petrushkina, Ekaterina A. Pigarova, Liudmila Ya. Rozhinskaya
}

Endocrinology Research Centre, Moscow, Russia

In this review, we discuss the main reasons for the vitamin D insufficiency in Russian Federation, as well as data on the prevalence of vitamin D deficiency among various population groups and regions, which confirm the widespread prevalence of vitamin D deficiency in the country. The discussed data suggest that the current vitamin D insufficiency in Russian population (reduced levels of $25(\mathrm{OH}) \mathrm{D}$ occurs in 50 - $94 \%$ of general population) is due to both a low level of its endogenous synthesis and insufficient intake from food : the territory of the country is located in a zone of low insolation, and at the same time, the main natural sources of vitamin D (sea fish of fatty varieties) and fortified with vitamin D products are very limited in the diet of the population. Taking measures to improve the status of vitamin $\mathrm{D}$ and maintaining the optimal serum levels of $25(\mathrm{OH}) \mathrm{D}$ in children and adults, adequate vitamin D intake will improve the condition of the musculoskeletal system, as well as reduce the risk of development and improve the control of some chronic diseases.

KEYWORDS: Vitamin D; recommended daily allowance; vitamin D deficiency; epidemiology

\section{ВОЗМОЖНОСТЬ ЭНДОГЕННОГО СИНТЕЗА ВИТАМИНА D}

Потребность в витамине D, поступающем с пищей и добавками, зависит от интенсивности воздействия на человека солнечных лучей. Эндогенный синтез витамина Dпроисходит при воздействии на кожуультрафиолетового (УФ) излучения спектра В (длина волны 290-315 нм). Облучение всего тела солнечным светом в дозе, соответствующей 1 минимальной эритематозной дозе (МЭД), т.е. минимальной дозой, вызывающей покраснение кожи через 24 ч после воздействия, у молодого человека эквивалентно приему 20000 ME витамина D 2 [1]. Однако в силу ряда причин указанный источник витамина D имеет крайне ограниченное значение в том числе в РФ. Прежде всего, территория страны расположена в зоне низкой инсоляции, т.е. интенсивность солнечного излучения невысока вследствие большого зенитного угла солнца, который дополнительно увеличивается в зимние месяцы, так что солнечные лучи фильтруются через озоновый слой под более косым углом. Поэтому в различных реги- онах страны синтез витамина D в коже не происходит от 4 до 6 месяцев в году $[1,2]$. Кроме того, количество УФ-излучения, доступного для синтеза витамина D, зависит от толщины слоя облаков и загрязненности атмосферы, в результате чего может увеличиваться время, необходимое для синтеза адекватного количества витамина D. Так, плотный слой облаков может снижать интенсивность солнечного излучения до 1\% от уровня, наблюдаемого при ясном небе, и даже рассеянные облака могут значимо снизить уровень УФ-излучения [3]. Вклад в снижение поступления витамина D в результате воздействия солнечного излучения может также вносить применение солнцезащитного крема (крем с SPF 8 снижает способность кожи к выработке витамина D более, чем на 95\%, а крем с SPF 15 - более чем на 98\% [4]), пожилой возраст (способность к выработке витамина D в коже снижается с возрастом по крайней мере в 3 раза [1]), смуглость кожи (может потребоваться в 3-5 раз более длительное пребывание под солнечными лучами для выработки аналогичного количества витамина D в сравнении со светлокожими людьми [5]), рекомендации по защите кожи 
от инсоляции при ряде заболеваний. Так, например, при воздействии на открытую поверхность кожи площадью 35\% (обследуемые лица были одеты в стандартную летнюю одежду, представленную футболками с коротким рукавом и шортами длиной до колен) УФ излучения, эквивалентного нахождению на солнце в ясный июньский полдень на широте г. Манчестер в течение 30 минут 3 раза в неделю, было достаточно, чтобы у 90\% взрослых со светлой кожей поддерживались уровни 25(OH)D выше 20 нг/мл, однако этого воздействия оказалось недостаточно для темнокожих участников исследования (V фототип по Фитцпатрику) [6]. Таким образом, при сочетании нескольких вышеперечисленных факторов количество витамина D, синтезируемого в коже под действием солнечного излучения, значительно снижается.

\section{ПОТРЕБЛЕНИЕ ВИТАМИНА D С ПИЩЕЙ}

В соответствии с клиническими рекомендациями Российской ассоциации эндокринологов, согласующимися с позицией Международного общества эндокринологов, рекомендуемая суточная норма потребления витамина D составляет не менее 600-800 ME для взрослых в возрасте 18-50 лет, не менее 800-1000 ME для лиц старше 50 лет, не менее 800-1200 ME для беременных и кормящих женщин (по мнению ряда экспертов - $2000 \mathrm{ME}$ в течение всей беременности [7]), при этом, согласно мнению ряда экспертов, для поддержания оптимальных уровней витамина D крови более 30 нг/мл может требоваться ежедневный прием более 1500-2000 ME в сутки, а при ожирении и нарушениях метаболизма витамина D более 6000-8000 МЕ в сутки [8, 9].

При этом весьма ограничены возможности получения витамина D с пищей. Наибольшее количество витамина D в форме витамина D3 содержится в жирной рыбе (консервированный тунец - 236 ME/100 г, лосось - 600-1000 ME/100 г дикого, 100-250 ME/100 г выращенного на ферме, консервированные сардины - 300-600 ME/100 г, консервированная макрель 250 ME/100 г), масле печени трески (400-1000 ME/1 ч.л.), яичных желтках (20 ME/шт). Источником витамина $\mathrm{D}_{2}$ могут являться некоторые грибы (в грибах шиитаке 100 ME/100 г свежих, 1600 ME/100 г сушеных), однако его содержание, как правило, непостоянно [8]. При этом по данным Федеральной службы государственной статистики за 2014 г., ежедневно потребляют рыбу лишь около $25 \%$ населения, $35 \%$ - 1 раз в неделю, 22,4 \% - несколько раз в месяц [10]. Таким образом, уровень потребления населением содержащих витамин D продуктов не обеспечивает должного поступления витамина D в организм.

\section{ФОРТИФИКАЦИЯ ПИЩЕВЫХ ПРОДУКТОВ}

Обогащенные продукты питания могут обеспечивать поступление большой части от необходимого количества витамина D. B CША и Канаде проводится обогащение витамином D молока, а также некоторых хлебобулочных изделий, апельсинового сока, готовых завтраков и молочных продуктов с типичным содержанием витамина D около $100 \mathrm{ME}$ на порцию, и 60\% всего полученного с пищей витамина D поступает из обогащенных пище- вых продуктов, в том числе 44\% - за счет молока [11, 12]. Однако, несмотря на это, в США по данным крупных эпидемиологических исследований потребление витамина D с пищей существенно ниже рекомендованной нормы [13]. В большинстве стран Европы в связи со случаями интоксикации у маленьких детей в 1950-е гг. была запрещена фортификация молока витамином $D$, однако в Швеции и Финляндии в настоящее время обогащается молоко, и в большинстве европейских стран добавляют витамин D в готовые завтраки, хлеб и маргарин [8]. B РФ фортификация продуктов витамином D проводится лишь на добровольной основе в связи с отсутствием законодательно закрепленной практики обогащения пищевых продуктов (по данным на 2013 г. обогащенные пищевые продукты, производимые $14 \%$ от общего числа предприятий, составили 5\% по объему производства [12]).

\section{ОБЕСПЕЧЕННОСТЬ НАСЕЛЕНИЯ ВИТАМИНОМ D И ПОСЛЕДСТВИЯ ЕГО ДЕФИЦИТА}

Диагностика дефицита витамина D, согласно действующим национальным, а также международным клиническим рекомендациям, основана на определении уровня 25(OH)D в сыворотке крови, и адекватными принято считать уровни 25(OH)D $\geq 30$ нг/мл; уровень 25(OH)D в сыворотке крови <20 нг/мл расценивается как дефицит витамина D, диапазон 20-29 нг/мл трактуется как недостаточная обеспеченность организма витамином [8, 9, 14]. В РФ проведен ряд исследований, результаты которых согласуются с мировыми данными и подтверждают повсеместную распространенность низких уровней витамина D среди населения страны (таблица 1).

Также выполнены исследования, направленные на оценку обеспеченности витамином D детской популяции. Крупнейшее исследование «РОДНИЧОК», посвященное распространенности дефицита витамина D среди детского населения в РФ, включило 1230 детей в возрасте от 1 месяца до 3 лет, наблюдающихся в 7 ведущих региональных исследовательских центрах, охватывающих территории Северо-Запада, Центра, Юга, Приволжья, Урала, Сибири и Дальнего Востока РФ. Результаты этого исследования показали, что достаточно обеспечен витамином D (что определяется как уровень 25(OH)D>30 нг/мл) лишь каждый третий ребенок первых 3 лет жизни. Наиболее высокая частота встречаемости недостаточных уровней витамина D (менее 30 нг/мл) отмечена во Владивостоке - у $88 \%$ детей, в Казани - у $83 \%$ детей, в Новосибирске - у $80 \%$ детей, в Ставрополе у 76\% детей, что связано с низкой частотой применения препаратов колекальциферола в этих регионах. Самая низкая частота дефицита витамина D зарегистрирована в Москве (58\%), Екатеринбурге (52\%) и Архангельске (55\%). При этом наибольший процент детей с достаточными уровнями витамина D отмечен среди детей в возрасте от 6 месяца до 1 года (59,6\%); среди детей старшего показано снижение процента обеспеченности витамином D (у детей 2-го года - 28,7\%, у детей 3-го года жизни лишь 13,2\%) [24]. Такие результаты обусловлены наибольшей частотой дополнительного приема препаратов колекальциферола детьми раннего возраста, проживающими в этих городах. Результаты этой рабо- 
Таблица 1. Средние уровни 25(OH)D и распространенность дефицита витамина D у населения PФ

\begin{tabular}{|c|c|c|c|}
\hline Регион & $\begin{array}{c}\text { Группа обсле- } \\
\text { дованных, } \\
\text { количество }\end{array}$ & Средний уровень 25(OH)D, нг/мл & $\begin{array}{c}\text { Количество лиц с выявленным } \\
\text { дефицитом витамина D } \\
\text { (уровень 25(OH)D < } 30 \text { нг/мл) }\end{array}$ \\
\hline $\begin{array}{l}\text { г. Санкт-Петербург } \\
\text { [15] }\end{array}$ & $\begin{array}{c}\text { взрослые и дети, } \\
\mathrm{n}=1011\end{array}$ & 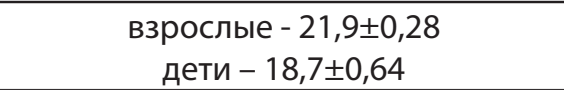 & $\begin{array}{c}\text { 82\% взрослых в возрасте 18-75 лет } \\
93 \% \text { детей 7-14 лет }\end{array}$ \\
\hline $\begin{array}{l}\text { г. Ростов-на-Дону } \\
{[16]}\end{array}$ & $\begin{array}{c}\text { взрослые и дети, } \\
\mathrm{n}=5335\end{array}$ & нет данных & $82,1 \%$ \\
\hline $\begin{array}{l}\text { Чувашская респу- } \\
\text { блика [17] }\end{array}$ & $\begin{array}{l}\text { молодые люди } \\
18-27 \text { лет, } \mathrm{n}=168\end{array}$ & $22,6 \pm 0,84$ & $94 \%$ \\
\hline $\begin{array}{l}\text { Амурская область } \\
{[18]}\end{array}$ & $\begin{array}{c}\text { дети, } \mathrm{n}=279, \\
\text { беременные } \\
\text { женщины, } \mathrm{n}=60\end{array}$ & 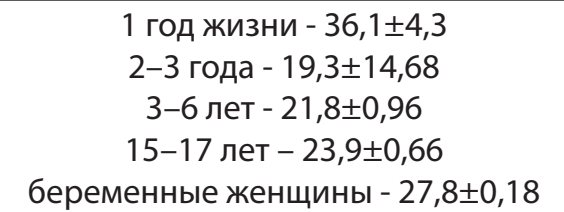 & $\begin{array}{c}\text { 72,6\% детей } \\
\text { 88,3\% подростков } \\
\text { 56,7\% беременных женщин }\end{array}$ \\
\hline $\begin{array}{l}\text { г. Санкт-Петербург } \\
\text { [19] }\end{array}$ & $\begin{array}{c}\text { беременные } \\
\text { женщины, } \mathrm{n}=205\end{array}$ & $\begin{array}{c}\text { от } 17,5 \pm 7,8 \text { до } 22,6 \pm 10,5 \text { нг/мл (в за- } \\
\text { висимости от времени года) }\end{array}$ & $\begin{array}{c}76,7-90 \% \\
\text { (в зависимости от времени года) }\end{array}$ \\
\hline г. Архангельск [20] & $\begin{array}{c}\text { взрослые, } \mathrm{n}=345 \\
\text { дети от } 0 \text { до } 15 \\
\text { лет, } \mathrm{n}=657\end{array}$ & $\begin{array}{c}\text { медианы: } \\
\text { дети до } 3 \text { лет - 27,7 } \\
\text { дети 6-7 лет - 13,1 } \\
\text { подростки } 13-15 \text { лет - 15,7 } \\
\text { студенты - 20,4 } \\
\text { взрослые - 24,3 }\end{array}$ & $\begin{array}{c}55 \text { \% детей до } 3 \text { лет } \\
91 \text { \% детей 6-7 лет } \\
99 \text { \% подростков } 13 \text { - } 15 \text { лет } \\
80 \text { \% студентов } \\
74 \text { \% взрослых }\end{array}$ \\
\hline $\begin{array}{l}\text { Республика Баш- } \\
\text { кортостан [21,22] }\end{array}$ & $\begin{array}{l}\text { взрослые стар- } \\
\text { ше } 50 \text { лет, } \mathrm{n}=188\end{array}$ & $\begin{array}{c}\text { в период минимальной инсоляции } \\
-13,42 \pm 0,51 \\
\text { в период максимальной инсоляции } \\
-22,84 \pm 0,6\end{array}$ & $\begin{array}{c}82 \% \text { - уровень } 25(\mathrm{OH}) \mathrm{D}<20 \text { нг/мл в пе- } \\
\text { риод минимальной инсоляции } \\
34 \% \text { - уровень } 25(\mathrm{OH}) \mathrm{D}<20 \text { нг/мл в пе- } \\
\text { риод максимальной инсоляции }\end{array}$ \\
\hline $\begin{array}{l}\text { Иркутская область } \\
\text { [23] }\end{array}$ & $\begin{array}{c}\text { взрослые и дети, } \\
\mathrm{n}=268\end{array}$ & 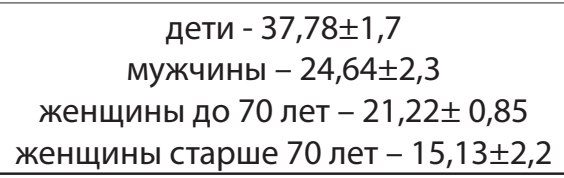 & $\begin{array}{c}\text { 48,8\% детей } \\
64,5 \% \text { мужчин } \\
82 \% \text { женщин до } 70 \text { лет } \\
100 \% \text { женщин старше } 70 \text { лет }\end{array}$ \\
\hline
\end{tabular}

ты согласуются с еще одним крупным исследованием, включившим детей различных возрастных категорий. При оценке уровней 25(OH)D у 1041 ребенка в возрасте от 1 месяца до 18 лет, проживающих в г. Москва, достаточный уровень 25(OH)D (>30 нг/мл) отмечался у небольшой части (26\% пациентов), в то время как у 74\% популяции выявлено снижение обеспеченности витамином D разной степени выраженности: у $28 \%$ выявлена недостаточность (20-29 нг/мл), у $33 \%$ - дефицит (<20 нг/мл), а у $13 \%$ - тяжелый дефицит витамина D (<10 нг/мл). Достаточный уровень витамина D отмечен только в группе пациентов первого года жизни (медиана уровня витамина D 34,2 нг/мл), в то время как уже с двухлетнего возраста у большой части исследуемых отмечается недостаточность витамина $D$, частота которой только нарастает с возрастом [25]. При обследовании в зимний период года 309 детей различных возрастных групп, проживающих в г. Казань, у детей раннего возраста (до 3 лет) сред-

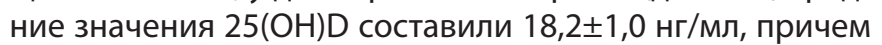
лишь у 14,8\% пациентов выявлены адекватные показатели 25(OH)D (более 30 нг/мл). 88,8\% детей школьного возраста имели сниженный уровень витамина D, при этом у $24 \%$ детей обеспеченность была на уровне выраженного дефицита (25(OH)D менее 10 нг/мл) [26]. В Якутии у здоровых детей и подростков (9-15 лет) зимой средний уровень витамина D составил 14 нг/мл, летом - 28,6 нг/мл, при этом зимой дефицит витамина D отмечается у $60 \%$ здоровых детей, летом - у 10\%; у 32,5\% здоровых детей регистрируется вторичный гиперпаратиреоз (среди детей с нарушениями осанки - у 62,4\%) [27]. У 140 здоровых детей и подростков, постоянно проживающих в средней полосе России, уровень 25(OH)D ниже 20 нг/мл в период максимальной инсоляции выявлен у 38,6\%; у 2,9\% выявлен выраженный дефицит витамина D (уровень $25(\mathrm{OH}) \mathrm{D}<8$ нг/мл) [28]. Среди 790 детей и подростков от 7 до 14 лет из Центрального и Северо-Западного регионов России уровни 25(OH)D составили в среднем $19,4 \pm 7,7$ нг/мл. Достаточная обеспеченность витамином D (25(OH)D>30 нг/мл) отмечена не более чем у 10\% детей, а выраженный дефицит $(25(\mathrm{OH}) \mathrm{D}<10$ нг/мл) был установлен у 8\% обследованных детей [29]. При обследовании 60 детей дошкольного возраста с частой респираторной заболеваемостью и 30 условно здоровых детей, составивших группу сравнения, у 59 детей (98,3\%) выявлено снижение уровня витамина D $(25(\mathrm{OH})$ $\mathrm{D}<30$ нг/мл), что в 2 раза чаще, чем у здоровых детей (43,3\%) [30]. В 2018 году опубликована созданная на основе проведенных эпидемиологических исследований национальная программа по коррекции недостаточности витамина D у детей и подростков Российской Федерации, которая предусматривает профилактический прием препаратов колекальциферола в дозе $1000 \mathrm{ME}$ в сутки у детей от 1 до 12 месяцев и от 3 до 18 лет, $1500 \mathrm{ME}$ в сутки у детей от 1 года до 3 лет, а для Европейского Севера России - 1000 МЕ в сутки у детей от 1 до 6 месяцев, 1500 ME в сутки у детей старше 6 месяцев [7]. 
Кроме того, ряд исследований посвящен оценке статуса витамина D у отдельных категорий лиц и подтверждает ассоциацию наличия дефицита витамина D с развитием и более тяжелым течением различных хронических заболеваний [31]. При обследовании 53 женщин с сахарным диабетом 2 типа выявлено значимое снижение 25(OH)D3 относительно контрольной группы $(20,44 \pm 0,6$ нг/мл vs. 30,86 $\pm 1,03$ нг/мл) [32]. В еще одном исследовании, включившем 57 пациенток с сахарным диабетом 2 типа в периоде постменопаузы, при определении у 15 пациенток уровня 25(OH)D средний уровень

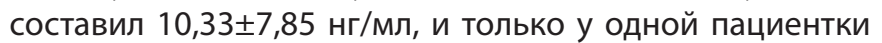
уровень 25(OH)D был на достаточном уровне (более 30 нг/мл), при этом повышение уровня паратгормона выше референсного интервала (15-65 пг/мл) выявлено у $21 \%$ пациенток [33]. При исследовании распространенности дефицита витамина D у больных псориазом, проживающих в Крымском регионе, в осенне-зимний период, средний уровень 25(OH)D среди 182 больных со-

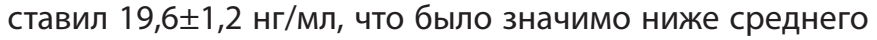
уровня 25(OH)D в контрольной группе (52,8土3,4 нг/мл). У $41 \%$ больных уровень 25(OH)D соответствовал выраженному дефициту витамина D (менее 10 нг/мл). Кроме того, большая распространенность поражения кожи была значимо ассоциирована с более низкими уровнями витамина D [34]. По результатам проведенного в Самарской области исследования, среди 46 взрослых пациентов с бронхиальной астмой (БА) более тяжелое течение заболевания было ассоциировано с более низкими уровнями витамина D: при интермиттирующей БА

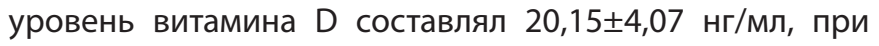

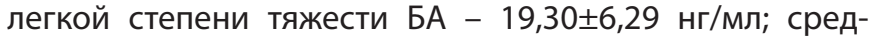
ней степени тяжести БА - 15,5 33,8 нг/мл; тяжелой БА $14,89 \pm 6,55$ нг/мл. В группе пациентов с контролируемой

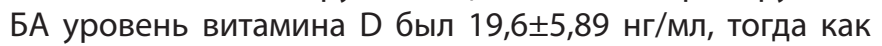
в группе с неконтролируемой БА - 15,3土4,27 нг/мл; у пациентов с дефицитом витамина D неконтролируемая БА диагностировалась в 4 раза чаще [35]. Среди 41 больного в возрасте от 18 лет до 27 лет, наблюдающегося по поводу депрессивного расстройства в г. Санкт-Петербург, уровни 25(OH)D более 30 нг/мл наблюдались лишь у 9\% обследованных лиц. Концентрация 25(OH)D ниже 10 нг/мл была выявлена у 29\% пациентов, среди которых в том числе были зарегистрированы все случаи крайне тяжёлых депрессивных расстройств [36]. У 123 обследованных пациентов с псориазом и псориатическим ар- тритом Иркутского региона средний уровень 25(OH)D составил 19,1 1,3 нг/мл, а уровни ниже 30 нг/мл были отмечены у 73,2\% [37]. При оценке концентрации 25(OH)D в сыворотке крови у 33 больных рассеянным склерозом в г. Санкт-Петербург, уровни ниже 30 нг/мл были выявлены у $96 \%$ пациентов [38].

Рекомендуемым препаратом для лечения дефицита витамина D является колекальциферол (D3). В РФ зарегистрированы лекарственные препараты витамина D в виде водных и масляных растворов. Фармакологические и физико-химические исследования показали, что для физиологического усвоения витамина D3 в тонком кишечнике необходимо участие желчных кислот, наиболее полно оно происходит из растворов так называемых «мицелл». Мицеллы - наночастицы с «жировой начинкой» (содержащей витамин D) и гидрофильной оболочкой, которая позволяет наночастицам равномерно распределяться по всему объему водного раствора, увеличивая всасывание и повышая биодоступность жирорастворимых витаминов A, D, E, К. Именно за счет образования мицелл и происходит «солюбилизация» витамина D.

Водный мицеллярный раствор холекальциферола $\left(\right.$ Аквадетрим ${ }^{\circledast}$ ) поступает в готовой для всасывания форме, обеспечивает хорошую степень всасывания витамина $\mathrm{D}$ в тонком кишечнике с минимальной зависимостью от состава диеты, приема лекарственных препаратов, состояния печени.

\section{ЗАКЛЮЧЕНИЕ}

Таким образом, в настоящее время доказана недостаточная обеспеченность витамином D как взрослого, так и детского населения РФ (сниженные уровни 25(OH)D выявляются в среднем у 80 \% лиц в общей популяции). Она может быть обусловлена как низким уровнем эндогенного синтеза витамина D вследствие недостаточной инсоляции, обусловленной географическим расположением территории страны и другими факторами, так и недостаточным потреблением его с пищей. Принятие мер по повышению статуса витамина D и поддержание оптимальной концентрации 25(OH)D в крови у детского и взрослого населения позволит улучшить состояние костно-мышечной системы, а также возможно снизить риск развития некоторых хронических заболеваний и смягчить их течение.

\section{СПИСОК ЛИТЕРАТУРЫ | REFERENCES}

1. Wacker M, Holick MF. Sunlight and Vitamin D. Dermatoendocrinol. 2014;5(1):51-108. doi: https://doi.org/10.4161/derm.24494

2. Webb AR, Kline L, Holick MF. Influence of Season and Latitude on the Cutaneous Synthesis of Vitamin D3: Exposure to Winter Sunlight in Boston and Edmonton Will Not Promote Vitamin D3Synthesis in Human Skin*. J. Clin. Endocr. Metab. 1988;67(2):373-378. doi: https://doi.org/10.1210/jcem-67-2-373

3. Engelsen O, Brustad M, Aksnes L, Lund E. Daily Duration of Vitamin D Synthesis in Human Skin with Relation to Latitude, Total Ozone, Altitude, Ground Cover, Aerosols and Cloud Thickness. Photochem. Photobiol. 2005;81 (6):1287. doi: https://doi.org/10.1562/2004-11-19-rn-375

4. Matsuoka LY, Ide L, Wortsman J, et al. Sunscreens suppress cutaneous vitamin D3 synthesis. J. Clin. Endocrinol. Metab. 1987; 64(6):1 165-1168. doi: https://doi.org/10.1210/jcem-64-6-1165
5. Clemens TL, Henderson SL, Adams JS, Holick MF. Increased Skin Pigment Reduces the Capacity of Skin to Synthesise Vitamin D3. The Lancet. 1982;319(8263):74-76. doi: https://doi.org/10.1016/s0140-6736(82)90214-8

6. Farrar MD, Kift R, Felton SJ, et al. Recommended summer sunlight exposure amounts fail to produce sufficient vitamin D status in UK adults of South Asian origin. Am. J. Clin. Nutr.. 2011;94(5):1219-1224. doi: https://doi.org/10.3945/ajcn.111.019976

7. Национальная программа "Недостаточность витамина D у детей и подростков Российской Федерации: современные подходы к коррекции" / Союз педиатров России [и др.]. - М.: Педиатръ, 2018. — 96 c. [The Union of pediatricians of Russia. "Insufficiency of vitamin D in children and adolescents of the Russian Federation: modern approaches to correction" national prorgam. Moscow: Pediatr; 2018. 96 p. (In Russ).] 
8. Holick MF, Binkley NC, Bischoff-Ferrari HA, et al. Evaluation, treatment, and prevention of vitamin D deficiency: An Endocrine Society Clinical Practice Guideline. J. Clin. Endocrinol. Metab. 2011;96(7):1911-1930. doi: https://doi.org/10.1210/jc.2011-0385

9. Пигарова Е.А., Рожинская Л.Я., Белая Ж.Е. и др. Клинические рекомендации Российской Ассоциации Эндокринологов по диагностике, лечению и профилактике дефицита витамина D у взрослых // Проблемы эндокринологии. - 2016. T. 62. - № 4. - C. 60-84. [Pigarova EA, Rozhinskaya LY, Belaya JE, et al. Russian Association of Endocrinologists recommendations for diagnosis, treatment and prevention of vitamin D deficiency in adults. Problems of Endocrinology. 2016;62(4):60-84. (In Russ.)] doi: https://doi.org/10.14341/probl201662460-84

10. Лайкам К.Э. Государственная система наблюдения за состоянием питания населения [Электронный ресурс]. 2014. Доступ по ссылke: http://www.gks.ru/free_doc/new_site/rosstat/smi/food_1-06_2. pdf [Laykam KE. Gosudarstvennaya sistema nablyudeniya za sostoyaniyem pitaniya naseleniya. Available from: http://www.gks.ru/ free_doc/new_site/rosstat/smi/food_1-06_2.pdf (In Russ.)]

11. Коденцова, В.М. Вржесинская О.А. Анализ отечественного и международного опыта использования обогащенных витаминами пищевых продуктов // Вопросы питания. - 2016. - Т. 85. № 2. - C. 31-50. [Kodentsova VM, Vrzhesinskaya OA. The analysis of domestic and international policy of food fortification with vitamins. Problems of Nutrition. 2016;85(2):31-50. (In Russ.)]

12. Коденцова В.М., Мендель О.И., Хотимченко С.А. и др. Физиологическая потребность и эффективные дозы витамина D для коррекции его дефицита. Современное состояние проблемы // Вопросы питания. - 2017. - Т. 86. - № 2. - C. 47-62. [Kodentsova VM, Mendel' OI, Khotimchenko SA, et al. Physiological needs and effective doses of vitamin D for deficiency correction. Current state of the problem. Problems of Nutrition. 2017;86(2):47-62. (In Russ.)]

13. Плещева А.В., Пигарова Е.А., Дзеранова Л.К. Витамин D и метаболизм: факты, мифы и предубеждения // Ожирение и метаболизм. - 2012. - T. 9. - № 2. - C. 33-42. [Plescheva AV, Pigarova EA, Dzeranova LK. Vitamin D and metabolism: facts, myths and misconceptions. Obesity and metabolism. 2012;9(2):33-42. (In Russ.)] doi: https://doi.org/10.14341/omet2012233-42

14. Пигарова Е.А., Петрушкина А.А. Терапевтические возможности коррекции дефицита витамина D у взрослых // Consilium Medicum. - 2018. - T. 20. - № 4. - C. 68-71. [Pigarova EA, Petrushkina AA. Treatment options of vitamin D deficiency in adults. Consilium Medicum. 2018;20(4):68-71. (In Russ.)] doi: https://doi. org/10.26442/2075-1753_2018.4.43-46

15. Каронова Т.Л., Гринева Е.Н., Никитина И.Л. и др. Уровень обеспеченности витамином D жителей Северо-Западного региона РФ (г. Санкт-Петербург и г. Петрозаводск) // Остеопороз и остеопатии. - 2013. - Т. 16. - № 3. - С. 3-7. [Karonova TL, Grinyova EN, Nikitina IL, et al. The prevalence of vitamin D deficiency in the northwestern region of the Russian Federation among the residents of St. Petersburg and Petrozavodsk. Osteoporosis and Bone Diseases. 2013;16(3):3-7. (In Russ.)] doi: https://doi.org/10.14341/osteo201333-7

16. Маркова Т.Н., Марков Д.С., Маркелова Т.Н. и др. Распространенность дефицита витамина D и факторов риска остеопороза у лиц молодого возраста // Вестник Чувашского Университета. - 2012. T. 234. - № 3. - C. 441-446. [Markova TN, Markov DS, Markelova $\mathrm{TN}$, et al. Prevalence of vitamin D deficiency and risk factors of the osteoporosis of young age persons. Vestnik Chuvashskogo universiteta. 2012;234(3):441-6. (In Russ.)]

17. Агуреева О.В., Жабрева Т.О., Скворцова Е.А. и др. Анализ уровня витамина D в сыворотке крови пациентов в Ростовской области // Остеопороз и остеопатии. - 2016. - Т. 19. - № 2. C. 47. [Agureeva OV, Zhabreva TO, Skvortsova EA, et al. Analiz urovnya vitamina D v syvorotke krovi patsientov v Rostovskoy oblasti. Osteoporosis and Bone Diseases. 2016;19(2):47. (In Russ.)] doi: https://doi.org/10.14341/osteo2016247-47

18. Борисенко Е.П., Романцова Е.Б., Бабцева А.Ф. Обеспеченность витамином D детского и взрослого населения Амурской области // Бюллетень. 2016. - Т. 9. - № 60. - C. 57-61. [Borisenko EP, Romancova EB, Babceva AF. Obespechennost' vitaminom D detskogo i vzroslogo naseleniya Amurskoj oblasti. Byulleten'. 2016;9(60):57-61. (In Russ.)]

19. Хазова Е.Л., Ширинян Л.В., Зазерская И.Е., и др. Сезонные колебания уровня 25-гидроксихолекальциферола у беременных, проживающих в Санкт-Петербурге // Гинекология. 2015. - Т.17. -
№ 4. - C. 38-42. [Khazova EL, Shirinyan LV, Zazerskaya IE, Bart VA Vasilieva EYu. Season fluctuations of level of 25-hydroxycholecalciferol in pregnant women living in Saint-Petersburg. Gynecology. 2015;17(4):38-42. (In Russ.)]

20. Малявская С.И., Кострова Г.Н., Лебедев А.В. Уровни витамина D у представителей различных групп населения города Архангельска // Экология человека. - 2018. - Т. 356. - № 1. - С. 60-64. [Malyavskaya SI, Kostrova GN, Lebedev AV, et al. 25(OH)D Levels in the Population of Arkhangelsk City in Different Age Groups. Ekologiya cheloveka. 2018; 356(1):60-64. (In Russ.)]

21. Нурлыгаянов Р.3., Сыртланова Э.Р. Распространённость дефицита витамина D у лиц старше 50 лет, постоянно проживающих в республике Башкоротостан, в период минимальной инсоляции // Остеопороз и остеопатии. - 2012. - Т.15. № 3. - C. 7-9. [Nurlygayanov RZ, Syrtlanova ER. Prevalence of vitamin $\mathrm{D}$ deficiency in people older than 50 years residing in the republic of Bashkortostan in periods of low insolation. Osteoporosis and Bone Diseases. 2012;15(3):7-9. (In Russ.)] doi: https://doi.org/10.14341/osteo201237-9

22. Нурлыгаянов Р.3., Сыртланова Э.Р., Минасов Т.Б. и др. Распространённость дефицита витамина D у лиц старше 50 лет, постоянно проживающих в республике Башкоротостан, в период максимальной инсоляции. 2015. - Т.18. - № 1. - С. 7-9. [Nurlygayanov RZ, Syrtlanov ER, Minasov TB, et al. The level of vitamin D in people over 50 years old residing in republic of Bashkortostan in the period of maximum insolation. Osteoporosis and Bone Diseases. 2015;18(1):7-9. (In Russ.)] doi: https://doi.org/10.14341/osteo201517-9

23. Спасич Т.А., Лемешевская Е.П., Решетник Л.А. и др. Гигиеническое значение дефицита витамина D у населения Иркутской области и пути его профилактики // Бюллетень ВСНЦ СО РАМН. 2014. - T. 100. - № 6. - C. 44-47. [Spasich TA, Lemeshevskaya EP, Reshetnik LA, et al. Gigienicheskoe znachenie deficita vitamina D u naseleniya Irkutskoj oblasti i puti ego profilaktiki. Byulleten' VSNC SO RAMN. 2014;100(6):44-47. (In Russ.)]

24. З Захарова И.Н., Мальцев С.В., Боровик Т.Э. и др. Результаты многоцентрового исследования "РОДНИЧОК" по изучению недостаточности витамина D у детей раннего возраста в России // Педиатрия. Журнал им. Г.Н. Сперанского. - 2015. - Т. 94. - № 1. C. 62-67. [Zaharova IN, Maltsev SV, Borovik TE, et al. Results of a multicenter research «Rodnichok» for the study of vitamin D insufficiency in infants in Russia. Pediatria. 2015;94 (1):62-67. (In Russ.)]

25. Захарова И.Н. Творогова Т.М., Соловьева Е.А. и др. Недостаточность витамина D у детей города Москвы в зависимости от сезона года // Практическая медицина. - 2017. - Т. 106. - № 5. C. 28-31. [Zakharova IN, Tvorogova TM, Solovjeva E.A, et al. Insufficiency of vitamin D in children in the city of Moscow depending on the year season. Practical medicine. 2017;106(5):28-31. (In Russ.)]

26. Мальцев С.В., Закирова А.М., Мансурова Г.Ш. Обеспеченность витамином D детей разных возрастных групп в зимний период // Российский вестник перинатологии и педиатрии. - 2017. - Т. 62. № 2. - C. 99-103. [Maltsev SV, Zakirova AM, Mansurova GS. Vitamin D provision in children of different age groups during the winter season. Rossiyskiy Vestnik Perinatologii i Pediatrii. 2017;62(2):99-103. (In Russ.)] doi: https://doi.org/10.21508/1027-4065-2017-62-2-99-103

27. Кривошапкина Д.М., Ханды М.В. Содержание витамина D в сыворотке крови у детей г. Якутска // Актуальные проблемы педиатрии. - 2006. - № S. - C. 295. [Krivoshapkina DM, Handy MV. Soderzhanie vitamina D v syvorotke krovi u detej g. Yakutska. Current pediatrics. 2016;(S):295. (In Russ.)]

28. Витебская А.В., Смирнова Г.Е., Ильин А.В. Витамин Д и показатели кальций- фосфорного обмена у детей, проживающих в средней полосе России, в период максимальной инсоляции // Остеопороз и остеопатии. - 2010. - Т. 13. - № 2. - C. 2-6. [Vitebskaya AV, Smirnova GE, Il'in AV. Vitamin D i pokazateli kal'tsiy-fosfornogo obmenau detey, prozhivayushchikh v sredney polose Rossii, $\checkmark$ periodmaksimal'noy insolyatsii. Osteoporosis and Bone Diseases. 2010;13(2):2-6. (In Russ.)] doi: https://doi.org/10.14341/osteo20102

29. Торшин И.Ю., Лиманова О.А., Сардарян И.С. и др. Обеспеченность витамином D детей и подростков 7-14 лет и взаимосвязь дефицита витамина D с нарушениями здоровья детей: анализ крупномасштабной выборки пациентов посредством интеллектуального анализа данных // Педиатрия. Журнал им. Г.Н. Сперанского. - 2015. T. 94. - № 2. - C. 175-184. [Torshin IYu, Limanova OA, Sardaryan IS, et al. Provision of vitamin D in children and adolescents aged 7 to 14 years and the relationship of deficiency of vitamin $D$ with violations 
of children's health: the analysis of a large-scale sample of patients by means of data mining. Pediatria. 2015;94(2):175-184. (In Russ.)]

30. Бабцева А.Ф., Романцова Е.Б., Борисенко Е.П. и др. Обеспеченность витамином D детей с частой респираторной заболеваемостью // Российский вестник перинатологии и педиатрии. 2016. - T. 61 - № 4. - C. 229-230. [Babceva AF, Romancova EB, Borisenko EP, et al. Obespechennost' vitaminom D detej s chastoj respiratornoj zabolevaemost'yu. Rossiyskiy Vestnik Perinatologii i Pediatrii. 2016;61(4):229-230. (In Russ.)]

31. Пигарова Е.А., Петрушкина А.А. Неклассические эффекты витамина D // Остеопороз и остеопатии. - 2017. - Т. 20. - № 3. C. 90-101. [Pigarova EA, Petrushkina AA. Non-classical effects of vitamin D. Osteoporosis and Bone Diseases. 2017;20(3):90-101. (In Russ.)] doi: https://doi.org/10.14341/osteo20173

32. Вербовой А.Ф., Шаронова Л.А., Капишников А.В., Демидова Д.В. Витамин D3, остеопротегерин и другие гормонально-метаболические показатели у женщин с сахарным диабетом 2 типа // Ожирение и метаболизм. - 2012. - Т. 9. - № 4. - С. 23-27. [Verbovoi AF, Sharonova LA, Kapishnikov AV, Demidova DV. Vitamin D3, osteoprotegerin and other hormonal and metabolic parameters in female patients with type 2 diabetes. Obesity and metabolism. 2012;9(4):23-27. (In Russ.)] doi: https://doi.org/10.14341/2071-8713-5125

33. Грачева Т.В., Лесняк О.М. Проблема вторичного гиперпаратиреоза и дефицит витамина Ду пациенток с сахарным диабетом 2 типа после менопаузы // Остеопороз и остеопатии. - 2016. - Т. 19. - № 2. - С. 56. [Gracheva TV, Lesnyak OM. Problema vtorichnogo giperparatireoza i defitsit vitamina D u patsientok s sakharnym diabetom 2 tipa posle menopauzy. Osteoporosis and Bone Diseases. 2016;19(2):56. (In Russ.)] doi: https://doi.org/10.14341/osteo2016256-56

34. Бекирова Э.Ю. Распространенность дефицита витамина D у больных псориазом, проживающих в Крымском регионе, в осен- не-зимний период // Вестник АГИУВ - 2013. - № 4 - С. 65-68. [Bekirova EYu. Rasprostranennost' deficita vitamina D u bol'nyh psoriazom, prozhivayushchih v Krymskom regione, v osenne-zimnij period. Herald ASIAME. 2013;(4):65-68. (In Russ.)]

35. Горемыкина М.С., Космынина М.А., Купаев В.И. Влияние витамина D на генез бронхиальной астмы в сочетании с метаболическим синдромом // Известия Самарского научного центра Российской академии наук. - 2014. - Т. 5. - № 2. - С. 776-778. [Goremykina MS, Kosmynina MA, Kupaev VI. Influence of vitamin D on the genesis of bronchial asthma in combination with metabolic syndrome. Izvestia of Samara Scientific Center of the Russian Academy of Sciences. 2014;5(2):776-778. (In Russ.)]

36. Дорофейков В.В., Задорожная М.С., Петрова Н.Н., и др. Дефицит витамина D у больных депрессивными расстройствами молодых лиц Санкт-Петербурга // Остеопороз и остеопатии. - 2016. - T. 19. - № 2. - C. 43-44. [Dorofejkov WV, Zadorozhnaya MS, Petrova NN, et al. Defitcit vitamina D u bol'nykh depressivnymi rasstroystvami u molodykh lits Sankt-Peterburga. Osteoporosis and Bone Diseases. 2016;19(2):43-44. (In Russ.)] doi: https://doi.org/10.14341/osteo2016243-44

37. Храмцова Н.А., Меньшикова Л.В., Грудинина О.В. Уровень 25(ОН) витамина D у пациентов с псориазом и псориатическим артритом // Остеопороз и остеопатии. - 2016. - Т. 19. - № 2. C. 46. [Khramtsova NA, Men'shikova LV, Grudinina OV. Uroven' $25(\mathrm{OH})$ vitamina D u patsientov s psoriazom i psoriaticheskim artritom. Osteoporosis and Bone Diseases. 2016;19(2):46. (In Russ.)] doi: https://doi.org/10.14341/osteo2016246-46

38. Шмонина И.А., Галкина О.В., Тотолян Н.А. и др. Уровень обеспеченности витамином D пациентов с рассеянным склерозом // Практическая медицина. - 2015. - Т. 90. - № 5. - C. 88-91. [Shmonina IA, Galkina OV, Totolyan NA, et al. Vitamin D status in patients with multiple sclerosis. Practical medicine. 2015;90(5):88-91. (In Russ.)]

\section{ИНФОРМАЦИЯ ОБ АВТОРАХ [AUTHORS INFO]}

*Петрушкина Александра Александровна [Alexandra A. Petrushkina, MD]; адрес: 117036, г. Moсква, ул. Дм. Ульянова, д.11 [address: 11, Dm. Ul'yanova str., Moscow, Russian Federation, 117036];

ORCID: http://orcid.org/0000-0002-7634-5457; eLibrary SPIN: 1970-2811; e-mail: a.petrushkina@yandex.ru

Пигарова Екатерина Александровна, к.M.H. [Ekaterina A. Pigarova, MD, PhD.];

ORCID: http://orcid.org/0000-0001-6539-466X; eLibrary SPIN: 6912-6331; e-mail: kpigarova@gmail.com

Рожинская Людмила Яковлевна, д.м.н., профессор [Liudmila Ya. Rozhinskaya, MD, PhD, Professor]; ORCID: http://orcid.org/0000-0001-7041-0732; eLibrary SPIN: 5691-7775; e-mail: rozhinskaya@rambler.ru

\section{ЦИТИРОВАТЬ:}

Петрушкина А.А., Пигарова Е.А., Рожинская Л.Я. Эпидемиология дефицита витамина D в Российской Федерации. // Остеопороз и остеопатии. — 2018. - Т. 21. — №3. — C.15-20. doi: https://doi.org/10.14341/osteo10038

\section{TO CITE THIS ARTICLE:}

Petrushkina AA, Pigarova EA, Rozhinskaya LYa. The prevalence of vitamin D deficiency in Russian Federation. Osteoporosis and bone diseases. 2018;21(3):15-20. doi: https://doi.org/10.14341/osteo10038 